\title{
Doğuştan çarpık ayakta alçılama sonrası aşilotomi ve tendon transferleri
}

\author{
Achilles tendon release and tibialis anterior tendon transfer \\ after primary casting in the treatment of congenital clubfoot
}

\author{
Bartu Sarısözen
}

Uludağ Üniversitesi Tıp Fakültesi, Ortopedi ve Travmatoloji Anabilim Dalı, Bursa

\begin{abstract}
Doğuştan çarpık ayağın Ponseti yöntemi ile tedavisi sırasında gelişen nükslerde veya tam düzelmemiş rezidüel deformitelerde gereken ikincil girişimlerin büyük çoğunluğu, Aşil tendon gevşetmeleri ve tibialis anterior tendon transferlerinden oluşmaktadır.

Gastrosoleus kas-tendon kompleksinde kısalık veya gerginliğin sonucu gelişen ekinus deformitesi bu sorunun hem başlangıcında, hem daha sonraki takibinde en çok sorun çıkaran ve en sık ek tedavi gerektiren patolojidir. Birincil alçılamadan sonra karşılaşılan ikinci sık sorun, ön ayağın dinamik supinasyon deformitesidir. Ekinus ve ön ayak supinasyonu izole veya birlikte olabilir. İki durumda da, bu deformitelerin tedavisinin geciktirilmesi, yapısal değişikliklerin eklendiği ve düzeltilmesi daha zor bir ayak deformitesine yol açar. Doğuştan çarpık ayağın tedavisi, hemen her zaman Ponseti alçılaması ile başlar ve sonraki nükslerde de alçılama tekrarlanır; ancak, bu iki deformitenin düzelmemesi durumunda gereken cerrahi girişimin geciktirilmemesi gerekir.
\end{abstract}

Anahtar sözcükler: çarpık ayak; doğumsal kusurlar; talipes ekinovarus; ekinus deformitesi; tendon transferi; tibialis anterior tendonu; çocuk
In the treatment of congenital clubfoot with Ponseti method, majority of secondary procedures which is used for relapses or residual deformities are composed of Achilles tendon release and tibialis anterior tendon transfer.

Equinus deformity caused by contracture or shortness of gastrocsoleus muscle-tendon complex is the most troublesome pathology of the early treatment period or subsequent follow-up that developes frequent complications and necessitates further treatment. The second most common problem encountered after the primary casting is the dynamic forefoot supination deformity. Equinus and forefoot supination may be isolated or co-existant. In both cases, delaying the treatment of these deformities leads to additional structural changes and then turns to a complex foot deformity which is more difficult to correct. Treatment of congenital clubfoot almost always starts with Ponseti casting, and recasting is the primary choice of treatment when deformity relapses. Nevertheless, if these deformities do not improve with casting, surgery should not be delayed.

Key words: clubfoot; congenital defects; talipes equinovarus; equinus deformity; tendon transfer; tibialis anterior tendon; children diyopatik doğuştan çarpık ayak (DÇA), Ponseti tekniği ile son derece başarılı bir biçimde tedavi edilebilmesine karşın, tedavi sonrasında gelişen nüks oranı az değildir. Çeşitli çalışmalarda nüks oranı \%3-54 arasında bildirmiştir. ${ }^{[1-6]}$ Nüks gelişmesi durumunda, bunun gecikmeden saptanması ve tedavi edilmesi, Ponseti yönteminin başarısı açısından çok önemlidir. ${ }^{[1,3,4]}$

DÇA'da nüks veya rezidü deformitelerle karşılaşılması durumunda, en sık gereken ikincil girişimler, Aşil tendonunun gevşetilmesi (ATG) ve tibialis anterior tendonunun transferi (TATT)'dir. Ponseti, alçılama yöntemi ile DÇA'nın erken tedavisi yapılırken ayak bileğinde $15-20^{\circ}$ 'den fazla dorsifleksiyon elde edilememesini, Aşil tendonuna girişimde bulunmak için yeterli gördüğünü belirtmişti. [1]

Dinamik ayak deformitelerinde, baskın olan kasların gevşetilmesi ve/veya ayakta başka bir noktaya transfer edilmeleri basit ve etkin bir girişimdir. DÇA'da gelişen rezidüel inversiyon deformitesinde uygulanan, tibialis anteriorun laterale transferi işlemi, deformitenin bir kere daha nüks etmesi riskini de önleyici özellik taşır.

- Illetişim adresi: Prof. Dr. Bartu Sarısözen, Uludağ Üniversitesi Tıp Fakültesi, Ortopedi ve Travmatoloji Anabilim Dalı, 16069 Bursa Tel: 0532 - 2570953 e-posta: bartu@uludag.edu.tr

- Geliş tarihi: 25 Mayıs 2015 Kabul tarihi: 25 Mayıs 2015 
Gereksiz süre kaybı, hareket edemeyen eklemlerle ilgili tüm kas gruplarının zaman içinde giderek daha da zayıflamalarına neden olur. Özellikle bebeğin en hızIı gelişim gösterdiği dönem olan 0-4 yaş aralığında, DÇA deformitesi olan bir ayakta, gelişimin önündeki engellerin çok gecikmeden kaldırılması önem taşır. $\mathrm{Bu}$ dönemde, DÇA'lı bir çocukta birincil tedaviden sonra gelişebilecek en sık ve önemli deformitelerden aşil tendon gerginliğine bağlı olan ekinus ve tibialis anterior tendon gerginliğine bağlı olan inversiyon, düzenli klinik kontrollerde düzelmiyor veya giderek kötüleşiyorsa, yaş ne olursa olsun girişimin geciktirilmemesi gerekir. Bazen ATG veya TATT'nin geciktirilmesi, daha sonra gerekecek girişimin basit bir tendon transferi veya gevşetmesi olmaktan çıkarak daha geniş çaplı bir yumuşak doku cerrahisine dönüşmesine, hatta buna osteotominin de eklenmesine neden olabilir.

\section{AŞiL TENDON GEVŞETILMESI (ATG)}

Çeşitli çalışmalarda, DÇA tedavisinde birincil alçılama sırasında gerekli olan ATG girişimi sıklığı çok değişen oranlarda bildirilmesine karşın, bu girişimin genelde hastaların yarısından çoğuna gerekebileceği düşünülmelidir. Haft ${ }^{[7]}$ bu oranı $\% 92$, Herzenberg ${ }^{[5]}$ $\% 91$, Morcuende ${ }^{[8]} \% 80$ ve Ponseti ${ }^{[9]} \% 79$ olarak bildirmişlerdir. ATG, Ponseti alçılama yönteminin ayrılmaz bir parçasıdır ve gerektiğinde uygulanması başarının anahtarıdır. Bebeğin birincil alçılama tedavisine başlama zamanına göre daha erken veya daha geç olabilmekle beraber, tipik bir hastada son alçılama ile birlikte yapılan ATG girişiminin zamanlaması, genellikle bebeğin 2. ayı civarına denk düşer.

Girişim sırasında Aşil tendonunun tam kesi ile gevşetilmesinden sonra, tendon kılıfi içinde oluşan boş kısmın dolması ve iyileşme hızı, bebeğin yaşı ile ilintilidir. Ortalama üç haftalık bir sürede, tendon bütünlügündeki eksik bölge dolar; ancak, tendon iyileşmesi ve yeniden şekillenme süresi iki yıla uzayabilir. ${ }^{[10]}$ Bu nedenle, ilk alçılama tedavisi sırasında ATG uygulanmışsa, daha sonra gelişen ekinus nükslerinin tedavisinde bu özellik göz önünde bulundurulmalıdır.

DÇA'da nüks veya rezidüel deformite nedeniyle ikincil tedavi gerektiren en önemli sorunların başında da ekinus deformitesi gelir. Ekinus deformitesi, yürüyüş bozukluğuna, denge sorunlarına, uzun dönemde ek ikincil deformitelerin gelişmesine ve ayak tabanının önünde nasır oluşumuna neden olur. Ayrıca, bunlara ek olarak ayak bileğinin hareket genişliğini önemli ölçüde kısıtlayarak, bu eklemi ilgilendiren kasların aktivitelerinin de kısıtlanmasına ve güçsüzleşmesine yol açar. Bu nedenlerle, idiyopatik DÇA tedavisi ve takibi sırasında saptandığı anda, önlem alınması gereken bir deformitedir.
DÇA tedavisinde, nüks veya rezidü saptanması durumunda, ikincil girişim olarak öncelikle alçılama yöntemi tekrarlanır. Ancak, gelişen nüks bir ekinus deformitesinde, Aşil tendonundaki kontraktür veya kısalık belirginse, tek başına alçılama çoğu kez yetersiz kalacaktır. Aynı birincil alçılama tedavisinde olduğu gibi, nüks varlığında da alçılama ile düzelmeyen ekinus deformitesinde ATG uygulanmalıdır. Özellikle birincil tedavinin takip döneminde olan, çoğu bir yaşına yaklaşmış veya daha büyük olan çocuklarda alçılama, yenidoğan dönemine oranla daha zor olacaktır. íkincil alçılama girişimlerine cevap vermeyen veya klinik değerlendirmesinde doğrudan ATG'ye karar verilen ayaklarda, Aşil tendonu tekrar perkütan tam kesi yapılarak gevşetilir. Birincil alçılama sırasında zaten ATG uygulanan ayaklarda, takip döneminde nüksle karşılaşıma oranı diğer ayaklara göre daha yüksektir. ${ }^{[11]}$ Bununla uyumlu olarak, başlangıçtaki alçılama öncesinde deformitesi daha sert olan, sınıflandırma sistemlerine göre deformite puanı daha yüksek olan ayaklarda, sonradan nüks gelişme oranı gibi, ATG oranları da daha yüksek olarak bulunmuştur. ${ }^{[12]}$

\section{ATG Teknik İpuçları}

Nüks ve rezidü durumunda öncelikle uygulanacak ATG, yöntem olarak Ponseti tarafından tanımlanan tekniktir. ATG, ameliyathane koşullarında ve sedatif analjezi ile birlikte yapılabileceği gibi, poliklinik koşullarında veya ofis ortamında da gerçekleştirilebilir. ${ }^{[13]}$ Perkütan ATG'ye alternatif olarak farklı tendon gevşetme teknikleri tanımlanmışsa da, hiç birisi perkütan yöntem kadar yaygınlaşmamıştır. ${ }^{[14-17]}$ Çeşitli durumlarda, bu teknikte değişiklik veya eklemeler yapma gereksinimi doğabilir. Özellikle, daha önce ATG uygulanmış bir ayakta, cilt ve altındaki dokularda yapışıklıklar gelişmiş olabilir. illk uygulama perkütan olmayıp kesiler yapılarak uygulandıysa, bu risk daha artar. Girişim öncesi, ayağın arteriyel dolaşımının değerlendirilmesi gereklidir. Yapısal varyasyonlara veya önceki işlemlerin izlerine bağlı olası bir dolaşım riski saptanıyorsa, seçenek olarak ATG'nin mini kesi veya kesiler kullanılarak yapılması düşünülmelidir. Çok sık olmamakla beraber, perkütan ATG sırasında gelişebilecek en önemli sorun, aşırı kanama ile karşılaşılmasıdır. Tendon kesisi sırasında en sık zedelenebilecek damar yapısı, lateralde yer alan peroneal arterdir. ${ }^{[18]}$ Daha az sıklıkla safen ven dalı veya nadiren tibialis posterior arteri de zedelenebilir. Nadiren görülen başka bir damarsal komplikasyon da zedelenme sonrası psödoanevrizma gelişmesidir. ${ }^{[19]}$ Böyle bir kanama ile karşılaşılması durumunda, alçının açılması, kompresif bandaj yapılarak kanamanın ve ayak dolaşımının takip edilmesi en uygun yaklaşımdır. Hemen her zaman, bu yaklaşımla kanama kontrol altına alınır ve daha ileri damarsal girişim gerekli olmaz. 
ATG ikincil tedavi yöntemi olarak uygulanıyorsa, tendinöz bölgenin sağlam kalmasını sağlayacak bir seçenek, gastrosoleusun kas-tendon bileşkesini kullanarak daha proksimal bir gevşetme yapılmasıdır. Park ve arkadaşları, ${ }^{[20]} 22$ ayakta rezidüel veya nüks ekinus deformitesinin tedavisi amacıyla Vulpius tekniğini kullanmışlar ve başarılı sonuç elde ettiklerini bildirmişlerdir.

ATG, izole ekinus deformitesi için uygulanıyorsa, alçının sonlandırılmasından sonra, çocuğun yaşı da göz önüne alınarak, ayak bileğinin plantar fleksiyona kaymasını engellemek için cihaz kullanımı tekrar gündeme alınmalıdır.

\section{TIBIALIS ANTERIOR TENDON TRANSFERI (TATT)}

DÇA'lı olguların yaklaşık \%20-25'inde TATT gerekir. Ponseti'nin kendi uzun dönem takibinde, sonradan TATT gereken olgu oranı \%46'dır. ${ }^{[1]}$ Birincil alçılamadan sonra gelişen nüks veya rezidü deformite durumlarında, tam olarak hangi ayaklarda TATT uygulanması gerektiği ile ilgili çok standartlaşmış ve kabul gören net kriterler yoktur. Genelde, yürüyüş sırasında saptanan dinamik rezidüel supinasyon deformitesi ana nedendir.

TATT, ön ayağın dinamik supinasyon ve adduksiyon deformitelerinde uygulanır. Bu deformitenin ana nedeni, tibialis anterior kasının baskınlığıdır. Dinamik deformite nitelemesi, ayakta adduksiyon, abduksiyon ve supinasyon, pronasyon eksenlerinde hareketlerin pasif olarak rahatlıkla yapılabilmesi anlamına gelir. Dolayısıyla, birincil alçılama sırasında düzeltilmeye çalışılan ön ayağın nispeten sert supinasyon ve adduksiyon deformitesinin aksine, TATT planlanan ayağın inversiyon deformitesi dinamik nitelikte olmalıdır. TATT planlanıyorsa unutulmaması gereken en önemli nokta, bu işlemin ayak deformitesinin yalnız dinamik komponentleri üzerinde etkili olacağıdır. Eğer ayakta sert ve pasif olarak düzeltilemeyen deformite komponentleri varsa, önce bunların düzeltilmesi ve arkasından TATT'nin eklenmesi, yöntemin başarısı için şarttır.

Birincil alçılama tedavisini bitirmiş ve hiçbir rezidüel deformitesi olmayan ayak ile kıyaslandığında, dinamik inversiyon deformitesi olan çarpık ayakta, ilgili eklemlerin hareket açıları daha azdır, eversiyon gücü daha düşüktür. ${ }^{[12]}$ Böyle bir ayak, supinasyon deformitesinin sonucunda, yürüyüş sırasında yerle daha az temas eder. Gray ve arkadaşları, ${ }^{[12]}$ temas alanının azlığına karşın arka ayağın ve ayağın lateral kısmının temas süresinin uzadığını bulmuşlardır. Dinamik inversiyon deformitesi, ayağın fonksiyonlarını etkilemek yanında ayakkabı uyumunu da zorlaştırır. Ancak tüm bu sorunların ötesinde, supinasyona neden olan kas yapılarının hep baskın kalmaları ve oluşturdukları kas dengesizliği, zaman içinde çarpık ayaktaki diğer deformite komponentlerinin de bir biçimde yinelemesine neden olabilir. Bu ise, başlangıçta dinamik olan deformitenin giderek sertleşmesine ve daha komplike olmasına yol açar.

Transferden sonra aşağıdaki gelişmelerin elde edilmesi beklenir:

a. Deformitenin düzelmesi.

b. İlerde gelişme riski olabilecek deformitenin önlenmesi.

c. Ayak bileği ekleminin daha düzgün bir anatomik pozisyon kazanması, dolayısıyla hareket açısının genişlemesi.

d. Ayak bilek hareketlerindeki artış sayesinde kas güçlerinde de artış olması. Özellikle evertör ve plantar-fleksör kasların güçlerinde sağlanan gelişmelerin asıl nedeni, artan eklem hareket açıklı̆̆ı sayesinde kas-tendon boylarının etkilenmesi ve artan iş gücü karşısında kurvetlenmeleridir.

e. Deformite tanımlamasında kullanılan Kite açıları gibi radyolojik ölçümlerde de düzelme olması.

Bu nedenlerle, DÇA tedavisinde özellikle ayak bilek hareketlerini kısıtlayan belirgin gerginlik veya kontraktürler varsa, düzeltilmeleri için uzun süre beklenmemelidir.

\section{TATT teknik ipuçları}

Nüks DÇA tedavisinde TATT uygulanması, ilk kez Garceau $^{[21]}$ tarafından tanımlandı. Bu orijinal teknikte, tendonun tümü tek parça halinde alınarak üç kesi (mediyal, anterior, lateral) ile 5. metatarsa nakledilmekteydi. Daha sonraları, aşırı düzelme riskini önlemek için, tendonun daha mediyale yakın noktalara transferi önerilmiştir. Örneğin; Palmer,, ${ }^{[22]}$ transfer için küboid kemiği kullanmıştır. Günümüzde genel uygulama, tendonun 3. küneiforma transfer edilmesidir. ${ }^{[23-29]}$ Tendon boyunun yetişmediği durumlarda veya aşırı düzelme ile ilgili bir ön kanı oluşuyorsa, 2. küneiform kemiğe de nakil yapılabilir. Aktarılan kemik hangisi olursa olsun, matkap ucu yardımıyla tendonun çapına uygun bir kanal açılır ve tendon dikişler yardımıyla bu kanaldan geçirilir. Son olarak, tendon dikişleri ayak tabanından çıkarılarak düğme üzerinde dikilir. Düğmenin ciltte sorun oluşturmaması ve gergin kalması için, cilt ile arasına tampon işlevi görecek sünger veya benzeri bir malzeme konarak desteklenir (Şekil 1).

Anterior tibial tendonun sadece bir yarısının transfer edildiği teknik (split transfer), başlangıçta spastik ayak deformiteleri için tanımlanmış olsa da, ${ }^{[26]}$ daha sonraları DÇA izleminde ortaya çıkan dinamik supinasyon deformitelerinde de kulllanılmaya başlamıştır. ${ }^{[24,25]}$ Tendon tek parça halinde ve tümüyle transfer edilecekse, ilk 

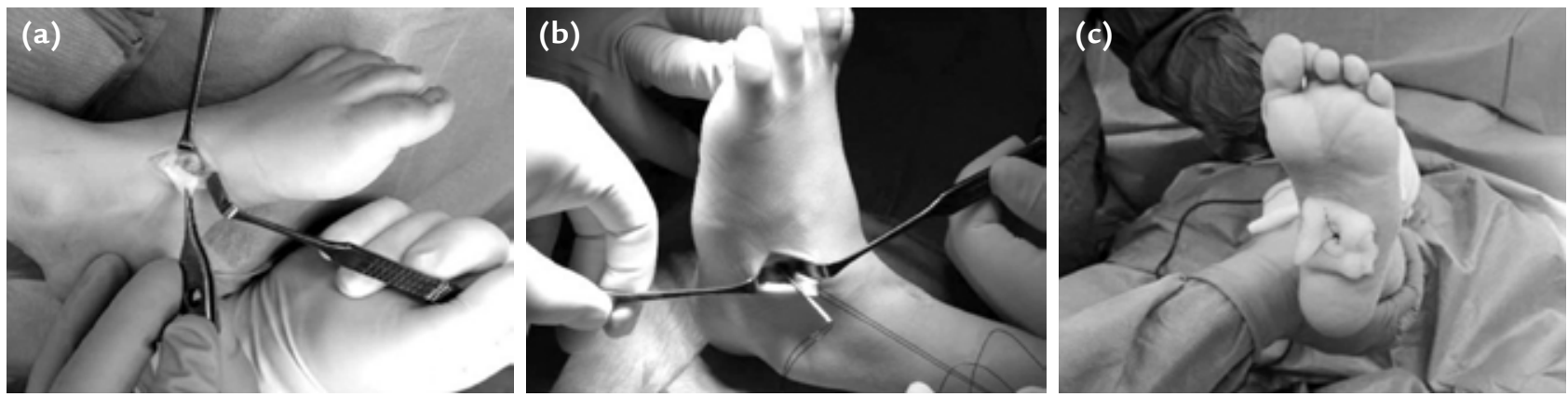

Şekil 1. a-c. Tibialis anterior tendon transferinin aşamaları: Mediyalde tendonun yapışma yerinden tek parça olarak kesilmesi ve serbestleştirilmesi (a), anteriorda ikinci bir kesi ile ayak bileğinin önüne alınan tendonun retinaküler bağların altından geçirilerek lateral yüzde 3. küneiforma nakledilmesi (b) ve tendon dikişlerinin ayak tabanında sünger ve düğme kullanılarak tespit edilmesi (c).

olarak mediyalden kesi ile tibialis anterior yapışma yeri bulunur ve tendon kesilerek serbestleştirilir. Daha sonra, bacağın ön yüzünde, ayak bileğinin hemen üzerinden küçük bir kesi ile tendon anteriora alınır ve takiben 3. bir kesi ile laterale transfer edilir. Tendon, ön yüzde eksplore edilmeden, yalnız iki kesi kullanılarak ve doğrudan cilt altından geçirilmek suretiyle de ayak dış yüzüne transfer edilebilir; bu şekilde teknik basitleşir ve kesi sayısı azalır, ancak tibialis anterior tendonunun yönelim ve yapışma açısı değişeceğinden, teorik olarak, transfer edildiği noktada yaratacağı çekme etkisinin daha az olması, bu yöntemin dezavantajıdır. ${ }^{[28]}$ Anteriorda kesi yapılmasının bir başka avantajı, tendonun ayak bileği önündeki bağların altından nakledilmesine olanak sağlamasıdır; ancak, bu yöntemde yapışıklık gelişme olasılığı, cilt altından kaydırmaya göre daha fazladır. Tam transfer uygulanan ayakların inversiyon hareket genişliğinin, yarım transfer uygulananlara göre daha az olduğu da öne sürülmüş̧tür. ${ }^{[24]}$

\section{ATG, TATT VE DIĞER GIRIŞiMLERIN BERABER UYGULANMALARI}

Birçok çocukta, ön ayağın dinamik supinasyon deformitesine, sert ekinus deformitesi de eşlik eder. Ekinus deformitesi ikincil alçılamalar ile düzeltilemiyorsa, TATT ile ATG işleminin beraber uygulanması gereklidir. Özellikle iki yaş ve üzerindeki çocuklarda, tek başına alçılama, düzeltmede yeterli olmayacaktır. Hatta deformitenin sertliği ve çocuğun daha da büyük olmasına bağlı olarak, bu iki yumuşak doku girişimine osteotomilerin de eklenmesi gerekli olabilir.

Ekinus deformitesinin düzeltilmesi yalnız Aşil tendonunun gevşetilmesi ile sağlanamazsa, kalkaneofibular bağ, ayak bileği eklemi ve/veya subtalar eklem arka kapsülü gibi yapıların da gevşetmeye dahil edilmesi gerekebilir. Supinasyon ve adduksiyon deformiteleri tamamen dinamik değilse ve gergin yapılar içeriyorsa, mediyalde de tendon ve kapsül gevşetmeleri eklenebilir. Unutulmaması gereken, dinamik olmayan veya bir başka deyişle sert komponentleri olan DÇA nüksünde, tek başına uygulanacak TATT'nin herhangi bir yarar sağlamayacağıdır.

\section{KAYNAKLAR}

1. Laaveg SJ, Ponseti IV. Long-term results of treatment of congenital club foot. J Bone Joint Surg Am 1980;62(1):23-31.

2. Morcuende JA, Dolan LA, Dietz FR, Ponseti IV. Radical reduction in the rate of extensive corrective surgery for clubfoot using the Ponseti method. Pediatrics 2004;113(2):376-80.

3. McKay SD, Dolan LA, Morcuende JA. Treatment results of late-relapsing idiopathic clubfoot previously treated with the Ponseti method. J Pediatr Orthop 2012;32(4):406-11. CrossRef

4. Ponseti IV, Campos J. The classic: observations on pathogenesis and treatment of congenital clubfoot. 1972. Clin Orthop Relat Res 2009;467(5):1124-32. CrossRef

5. Herzenberg JE, Radler C, Bor N. Ponseti versus traditional methods of casting for idiopathic clubfoot. J Pediatr Orthop 2002;22(4):517-21.

6. Dietz FR. Treatment of a recurrent clubfoot deformity after initial correction with the Ponseti technique. Instr Course Lect 2006;55:625-9.

7. Haft GF, Walker CG, Crawford HA. Early clubfoot recurrence after use of the Ponseti method in a New Zealand population. J Bone Joint Surg Am 2007;89(3):487-93.

8. Morcuende JA, Abbasi D, Dolan LA, Ponseti IV. Results of an accelerated Ponseti protocol for clubfoot. J Pediatr Orthop 2005;25(5):623-6.

9. Ponseti IV, Smoley EN. The classic: congenital club foot: the results of treatment. 1963. Clin Orthop Relat Res 2009;467(5):1133-45. CrossRef

10. Niki $H$, Nakajima $H$, Hirano $T$, Okada $H$, Beppu $M$. Ultrasonographic observation of the healing process in the gap after a Ponseti-type Achilles tenotomy for idiopathic congenital clubfoot at two-year follow-up. J Orthop Sci 2013;18(1):70-5. CrossRef

11. Dobbs MB, Rudzki JR, Purcell DB, Walton T, Porter KR, Gurnett CA. Factors predictive of outcome after use of the Ponseti method for the treatment of idiopathic clubfeet. J Bone Joint Surg Am 2004;86-A(1):22-7. 
12. Gray K, Burns J, Little D, Bellemore M, Gibbons P. Is tibialis anterior tendon transfer effective for recurrent clubfoot? Clin Orthop Relat Res 2014;472(2):750-8. CrossRef

13. Lebel E, Karasik M, Bernstein-Weyel M, Mishukov Y, Peyser A. Achilles tenotomy as an office procedure: safety and efficacy as part of the Ponseti serial casting protocol for clubfoot. J Pediatr Orthop 2012;32(4):412-5. CrossRef

14. Cummings RJ. The effectiveness of botulinum $A$ toxin as an adjunct to the treatment of clubfeet by the Ponseti method: a randomized, double blind, placebo controlled study. J Pediatr Orthop 2009;29(6):564-9. CrossRef

15. Dogan A, Uzumcugil O, Sarisozen B, Ozdemir B, Akman YE, Bozdag E, Sunbuloglu E, Bozkurt E. A comparison of percutaneous and mini-open techniques of Achilles tenotomy: an experimental study in rats. J Child Orthop 2009;3(6):48591. CrossRef

16. Minkowitz B, Finkelstein BI, Bleicher M. Percutaneous tendoAchilles lengthening with a large-gauge needle: a modification of the Ponseti technique for correction of idiopathic clubfoot. J Foot Ankle Surg 2004;43(4):263-5.

17. Sharma S, Butt MF, Singh M, Sharma S. The posterior to anterior controlled technique of percutaneous Achilles tenotomy in the correction of idiopathic clubfoot: a technical report. J Pediatr Orthop B 2013;22(3):249-51. CrossRef

18. Dobbs MB, Gordon JE, Walton T, Schoenecker PL. Bleeding complications following percutaneous tendoachilles tenotomy in the treatment of clubfoot deformity. J Pediatr Orthop 2004;24(4):353-7.

19. Burghardt RD, Herzenberg JE, Ranade A. Pseudoaneurysm after Ponseti percutaneous Achilles tenotomy: a case report. J Pediatr Orthop 2008;28(3):366-9. CrossRef

20. ParkSS, Lee HS, Han SH, ParkJW, de Peralta MJ. Gastrocsoleus fascial release for correction of equinus deformity in residual or relapsed clubfoot. Foot Ankle Int 2012;33(12):1075-8. CrossRef
21. Garceau GJ. Anterior tibial tendon transposition in recurrent congenital club-foot. J Bone Joint Surg Am 1940;22:932-6.

22. Garceau GJ, Palmer RM. Transfer of the anterior tibial tendon for recurrent club foot. A long-term follow-up. J Bone Joint Surg Am 1967;49(2):207-31.

23. Ezra E, Hayek S, Gilai AN, Khermosh O, Wientroub S. Tibialis anterior tendon transfer for residual dynamic supination deformity in treated club feet. J Pediatr Orthop B 2000;9(3):207-11.

24. Kuo KN, Hennigan SP, Hastings ME. Anterior tibial tendon transfer in residual dynamic clubfoot deformity. J Pediatr Orthop 2001;21(1):35-41.

25. Lampasi M, Bettuzzi C, Palmonari M, Donzelli O. Transfer of the tendon of tibialis anterior in relapsed congenital clubfoot: long-term results in 38 feet. J Bone Joint Surg $\mathrm{Br}$ 2010;92(2):277-83. CrossRef

26. Hoffer MM, Reiswig JA, Garrett AM, PerryJ. The split anterior tibial tendon transfer in the treatment of spastic varus hindfoot of childhood. Orthop Clin North Am 1974;5(1):31-8.

27. Masrouha KZ, Morcuende JA. Relapse after tibialis anterior tendon transfer in idiopathic clubfoot treated by the Ponseti method. J Pediatr Orthop 2012;32(1):81-4. CrossRef

28. Knutsen AR, Avoian T, Sangiorgio SN, Borkowski SL, Ebramzadeh E, Zionts LE. How do different anterior tibial tendon transfer techniques influence forefoot and hindfoot motion? Clin Orthop Relat Res 2015;473(5):1737-43. CrossRef

29. Farsetti P, Caterini R, Mancini F, Potenza V, Ippolito E. Anterior tibial tendon transfer in relapsing congenital clubfoot: long-term follow-up study of two series treated with a different protocol. J Pediatr Orthop 2006;26(1):83-90. 